\title{
Musculoskeletal imaging at 3T: imaging and optimization
}

\author{
James S. Meyer
}

Published online: 17 June 2008

(C) Springer-Verlag 2008

Erratum to: Pediatr Radiol (2008) 38 (Suppl 2): S243-S245

DOI 10.1007/s00247-008-0768-2

There were errors in the last term of each equation: $\left(e^{-\mathrm{TR} / \mathrm{T} 2}\right)$ in (1) and $\left(\mathrm{e}^{-\mathrm{TR} / \mathrm{T} 2^{*}}\right)$ in (2); the corrected equations below have TE instead of TR.

Corrected equations:

$\mathrm{SNR}_{\mathrm{SE}} \propto \mathrm{B}_{0} \mathrm{~V}\left(\left(\mathrm{~N}_{\mathrm{PE}} \mathrm{N}_{\mathrm{PA}} \mathrm{N}_{\mathrm{AV}}\right) / \mathrm{BW}\right)^{1 / 2}\left(1-\mathrm{e}^{-\mathrm{TR} / \mathrm{T} 1}\right) \mathrm{e}^{-\mathrm{TE} / \mathrm{T} 2}$

$$
\begin{aligned}
& \mathrm{SNR}_{\mathrm{GRE}} \propto \mathrm{B}_{0} \mathrm{~V}\left(\left(\mathrm{~N}_{\mathrm{PE}} \mathrm{N}_{\mathrm{PA}} \mathrm{N}_{\mathrm{AV}}\right) / \mathrm{BW}\right)^{1 / 2} \\
& \sin (\theta)\left(1-\mathrm{e}^{-\mathrm{TR} / \mathrm{T} 1}\right) /\left(1-\mathrm{e}^{-\mathrm{TR} / \mathrm{T} 1} \cos (\theta)\right) \mathrm{e}^{-\mathrm{TE} / \mathrm{T} 2 *}
\end{aligned}
$$

The online version of the original article can be found at http://dx.doi. org/10.1007/s00247-008-0768-2.

J. S. Meyer $(\bowtie)$

Children's Hospital of Philadelphia,

Philadelphia, PA, USA

e-mail: meyer@email.chop.edu

J. S. Meyer

University of Pennsylvania School of Medicine,

Philadelphia, PA, USA 